\title{
O que dizem as propostas curriculares do Brasil sobre o tema saúde e as doenças negligenciadas?: aportes para a educação em saúde no ensino de ciências
}

\author{
What's written in Brazil's curriculum proposals about health \\ and neglected diseases?: contributions towards health education \\ in science education
}

Sheila Soares de Assis ${ }^{1}$. Tania Cremonini Araujo-Jorge ${ }^{2}$

\begin{abstract}
Resumo: As doenças negligenciadas correspondem a um grupo de agravos de relevância nacional e internacional. O tema das doenças negligenciadas precisa transcender o setor da saúde e sua abordagem deve estar presente também no ensino. Além disso, o controle e a prevenção destes agravos, bem como a saúde, necessitam ser compreendidos como um fenômeno multifacetado. Analisamos o tema das doenças negligenciadas nas propostas curriculares dos estados brasileiros destinados à disciplina de Ciências do $6^{\circ}$ ao $9^{\circ}$ ano do ensino fundamental. Reunimos ao todo 24 propostas curriculares. Os resultados apontaram que somente nove destes currículos abordaram as doenças negligenciadas como conteúdos de relevância e que persiste ainda a ideia de que a saúde é um bem restritamente biológico ou produto de ações prescritivas e ganhos cognitivos.
\end{abstract}

Palavras-chave: Doenças negligenciadas. Parâmetros curriculares nacionais. Currículo Ensino de ciências. Educação em saúde. Ensino fundamental.

\begin{abstract}
Neglected diseases are of national and international relevance. The neglected diseases' subject-matter must transcend the health sector and its approach must be present in education as well. In addition, the control and prevention of these diseases, as well as health, should be understood as a multifaceted phenomenon. This study aimed to analyze the neglected diseases' theme in the Brazilian states' curriculum proposals for elementary school's Science subject from 6th to 9th grade. A total of 24 curriculum proposals were gathered and examined. The results showed that only nine of these curricula dealt with neglected diseases as relevant content, still allowing the idea to persist that health is a strictly a biological good or a product of prescriptive actions and cognitive gains.
\end{abstract}

Keywords: Neglected diseases. Government curriculum proposals. Curriculum Science Education. Health education. Elementary School.

\footnotetext{
${ }^{1}$ Fundação Oswaldo Cruz (Fiocruz), Rio de Janeiro, RJ, Brasil. E-mail: <sheila.assisbiouff@gmail.com>.

${ }^{2}$ Fiocruz, Instituto Oswaldo Cruz (IOC), Laboratório de Inovações em Terapias, Ensino e Bioprodutos (LITEB), Rio de Janeiro, RJ, Brasil. Orcid: <https://orcid.org/0000-0002-8233-5845>.
} 


\section{Introdução}

Segundo a Organização Mundial da Saúde, o grupo de doenças que afeta e se perpetua, predominantemente, junto às populações em condição de pobreza ou extrema pobreza, são consideradas negligenciadas (WORLD HEALTH ORGANIZATION, 2012). A dengue, leishmanioses, leptospirose, tuberculose, doença de Chagas e hanseníase são exemplos de doenças que compõem esse grupo e que figuram como extremamente relevantes no Brasil, dada sua ocorrência no território nacional. Para a estruturação de ações de prevenção e controle das doenças negligenciadas, deve-se considerar, no âmbito da formulação das políticas públicas, a diversidade de condicionantes tais como características biológicas, econômicas, sociais, ambientais, culturais, entre outras (WORLD HEALTH ORGANIZATION, 2015).

De modo geral, as doenças negligenciadas compõem um ciclo de desigualdade e o retroalimentam (WORLD HEALTH ORGANIZATION, 2007). Este cenário não se restringe ao nível financeiro, embora este exerça um importante papel para essa configuração. No Brasil, iniciativas têm sido implantadas, principalmente na última década, visando à redução das doenças negligenciadas e à construção de um cenário mais equânime. Porém, o país continua sendo a nação das Américas com maior carga proveniente das doenças negligenciadas (NEGLECTED..., 2014).

Desde os primórdios de sua conceituação, a pobreza esteve relacionada a situações de insuficiência de recursos, múltiplas privações, exclusão e desigualdade de classes. Como resultante, os indivíduos imersos nesta realidade não possuem condições básicas de subsistência e ainda de acesso aos serviços essenciais como saúde e educação (PARDO, 2007). A pobreza constitui uma questão social em que são produzidas e reproduzidas desigualdades no nível social, político, econômico e cultural. Desta forma, abordá-la transcende as circunstâncias econômicas, sociais e políticas (YAZBEK, 2012). Assim, a questão das doenças vinculadas a esse contexto deve ultrapassar as ações simplistas e de cunho individual. Exige-se, então, um conjunto de ações articuladas de diferentes níveis (BRICEÑO-LEÓN, 2005). Portanto, o ensino formal não pode ser ignorado, visto a potencialidade de sua contribuição para construção do pensamento crítico. Além disso, uma das falhas identificadas que interferem no controle das doenças negligenciadas é ausência de formação cidadã. Esta lacuna corresponde a "[...] baixa eficiência das ações educativas para a formação de sujeitos críticos que possam atuar sobre sua condição de saúde [...] através do cumprimento de seus deveres e ciência sobre seus direitos" (ASSIS, 2012, p. 23).

Em suma, a negligência atribuída aos agravos em saúde não se restringe a um único nível (o nível do setor da saúde), mas este cenário é sustentado por uma série de fatores que permitem sua ocorrência e perpetuação. No âmbito do ensino, é imprescindível que se coloque em pauta a temática, tendo em vista que a escola deve se ocupar em abordar questões científicas de forma contextualizada com a realidade.

\section{Doenças negligenciadas, promoção da saúde e o ensino formal}

O espaço escolar manifesta-se como instituição de reprodução, proposital ou não, das aspirações da ordem vigente na sociedade no processo de construção de conhecimento. Neste contexto, Krasilchik (2000) aponta que o ensino de Ciências é modificado em função do âmbito 
político-econômico, tanto nacional como internacional. A abordagem dos temas relacionados à saúde tem se apresentado na escola de forma diversificada seguindo esta tendência.

Atualmente, a saúde é alocada como um tema transversal. Indica-se, assim, que as questões sobre esta temática devam ser tratadas em todas as disciplinas do currículo. Apesar disso, ao longo do tempo, diversos trabalhos têm indicado que mesmo havendo uma recomendação de uma abordagem transversal para o tema, na prática escolar presente no contexto nacional, a saúde continua centrada nas disciplinas de Ciências e Biologia (ASSIS, 2012; ASSIS; ARAÚJOJORGE, 2014a; MOHR, 2009; PINHÃO; MARTINS, 2012).

Soma-se a isso o fato de que, na atualidade, busca-se o desenvolvimento de ações sobre a saúde que transponham o nível curativo. Almeja-se desta forma que na escola as práticas possam contribuir com o desenvolvimento da autonomia e do senso crítico dos alunos, cooperando, de tal modo, para a formação de sujeitos capazes de agir sob sua condição de vida e, consequentemente, aptos a preservar a saúde individual e coletiva. Essa concepção concorre com a perspectiva do letramento científico que prevê uma integração dos conteúdos e não sua fragmentação (SANTOS, 2007). Neste sentido, Krasilchik (1987) alerta para o fato da ausência de vínculo entre os conteúdos escolares e a realidade dos alunos. Segundo a autora, essa limitação faz com que a disciplina se torne irrelevante e sem significado, à medida que não se baseia no conhecimento dos alunos e não parte de seu universo de interesses. Considerar que as doenças negligenciadas consistem em um grupo de agravos presente na vida dos brasileiros e advogar que o tema deva estar presente nas salas de aulas é o mesmo que buscar a superação deste modelo de ensino descontextualizado da realidade.

No Brasil e no mundo têm sido realizados diversos trabalhos relacionando o espaço escolar e as doenças negligenciadas. Estes se referem, principalmente, a inquéritos parasitológicos envolvendo o público escolar, administração de tratamento medicamentoso e ainda ações em torno do levantamento de conhecimentos, atitudes e práticas com a finalidade de subsidiar os programas de prevenção e controle de agravos voltados às populações específicas (FAVRE et al., 2009; HAN et al., 2014; LESLEY et al., 2015; NICOLAY et al., 2015). Os estudos concordam sobre a importância da abordagem do tema das doenças negligenciadas no espaço escolar, visto que além de se ter na escola uma parcela representativa da população de determinada localidade, há também o envolvimento da família no processo. Deste modo, a escola se torna um local de multiplicação/construção de saberes e para a materialização de políticas com a finalidade de promoção da saúde em conjunto com a prevenção e o controle de agravos.

Em trabalho anterior, refletimos sobre o tema das doenças negligenciadas em propostas curriculares de cinco estados brasileiros. Verificamos que a abordagem da temática parece incipiente e indicamos a relevância da análise das propostas curriculares com a finalidade de elucidar como essa importante questão tem sido incluída no espaço escolar (ASSIS; ARAÚJO-JORGE, 2014a). Já em outro trabalho propusemos o cotejamento entre as propostas curriculares de Ciências e documentos oficinais do Programa Saúde na Escola (PSE). A finalidade do estudo foi confrontar as contribuições de ambos os campos, a convergência, bem como a divergência de ideias sobre as doenças negligenciadas. Por fim, concluimos que as diretrizes do PSE são construídas sem que se considere as propostas curriculares e que estes dois eixos possuem pouca articulação entre si no que se refere à temática em questão (ASSIS; ARAÚJO-JORGE, 2014b).

Ressaltamos que o caráter atribuído às doenças negligenciadas e até o tempo destinado ao tema no espaço escolar relaciona-se com a visão de saúde incorporada nas propostas curri- 
culares e a importância creditada à questão. As propostas curriculares são documentos que se caracterizam como um produto onde se expressa uma cadeia de conhecimentos socialmente valorizados. Ele pode ser concebido ainda como um artefato social e cultural, fruto de demandas sociais. Implica em relações de poder, reproduz visões de mundo, produz identidades individuais e sociais (BRITO; FREITAS, 2009; GOODSON, 1995).

Dada a emergência da temática das doenças negligenciadas no contexto nacional e sua relevância no âmbito escolar, este trabalho pretende expandir o debate já travado. Assim sendo, propusemos uma análise do tema das doenças negligenciadas e a perspectiva de saúde apresentada nas propostas curriculares dos estados brasileiros destinados à disciplina de Ciências do $6^{\circ}$ ao $9^{\circ}$ ano do ensino fundamental.

\section{Metodologia}

O trabalho contemplou a metodologia qualitativa, com base na análise documental. A técnica foi eleita por possibilitar um olhar exploratório em relação a um problema de pesquisa que visa ser aprofundado. A análise documental permite que sejam evidenciados fatos novos a respeito do objeto de pesquisa e que se complementem as informações já existentes (ALVES -MAZZOTTI; GEWANDSZNAJDER, 1998; BOGDAN; BIKLEN, 1994).

Inicialmente, realizamos o levantamento das propostas curriculares por meio do acesso ao site das secretarias estaduais de educação. Quando o documento não foi localizado na versão eletrônica realizamos o contato via e-mail, correspondência impressa dirigida as secretarias de educação e contato telefônico. Ao todo, foram reunidos 24 documentos (Quadro 1) ${ }^{3}$.

Não foi possível ter acesso aos documentos de três Estados (Amazonas, Pará e Rio Grande do Norte) por ausência de resposta das respectivas secretarias ou por indicarem que o documento se encontrava em fase de reformulação, não havendo prazo para finalização do processo. Os documentos foram coletados no período de dezembro de 2013 a maio de 2015 e todos foram indicados como utilizados no ano letivo de 2014.

Após a coleta, o material foi submetido à leitura exaustiva. Em seguida foi realizada uma nova leitura orientada pelas seguintes questões: (1) Qual/quais perspectiva (s) de saúde considerada no documento?; (2) $\mathrm{O}$ documento abordou as doenças negligenciadas em algum momento?; (3) Se sim, qual delas e de que forma?; (4) Quais conteúdos relacionados à saúde são indicados?; e, (5) Qual a abordagem em relação à saúde é proposta?

A análise realizada foi delimitada seguindo uma perspectiva interpretativa em que possibilita ao pesquisador explorar o sentido que nem sempre está explícito no texto (DEMO, 1995). O exercício de análise se centra no esforço de interpretação do pesquisador (MINAYO, 2010).

\footnotetext{
${ }^{3}$ Os documentos encaminhados pelas Secretarias Estaduais de Educação do Rio Grande do Sul, Ceará, Tocantins, Roraima e Minas Gerais não foram disponibilizados on-line e sim remetidos diretamente as pesquisadoras após solicitação formal. Em dezembro de 2017, durante a revisão final deste artigo, foi feita nova consulta ao site dos estados do Amazonas, Pará e Rio Grande do Norte, confirmando a ausência das propostas curriculares ainda neste ano.
} 
Quadro 1. Região, estado e ano de produção das propostas curriculares analisadas

\begin{tabular}{|c|c|c|}
\hline Região & Estado & Ano de publicação \\
\hline \multirow{7}{*}{ Norte } & Acre & 2010 \\
\hline & Rondônia & 2013 \\
\hline & Tocantins & 2009 \\
\hline & Amapá & 20--? \\
\hline & Roraima & 2010 \\
\hline & Amazonas & $\mathrm{s} / \mathrm{a}$ \\
\hline & Pará & $\mathrm{s} / \mathrm{a}$ \\
\hline \multirow{9}{*}{ Nordeste } & Pernambuco & 2013 \\
\hline & Alagoas & 2010 \\
\hline & Bahia & [2018] \\
\hline & Piauí & 2013 \\
\hline & Ceará & 20--? \\
\hline & Paraíba & 2013 \\
\hline & Sergipe & 2011 \\
\hline & Maranhão & 2014 \\
\hline & $\begin{array}{l}\text { Rio Grande do } \\
\text { Norte }\end{array}$ & $\mathrm{s} / \mathrm{a}$ \\
\hline \multirow{4}{*}{ Centro-oeste } & Distrito Federal & $\mathrm{s} / \mathrm{d}$ \\
\hline & Goiás & 20--? \\
\hline & Mato Grosso & 2010 \\
\hline & Mato Grosso do Sul & 2012 \\
\hline \multirow{4}{*}{ Sudeste } & Rio de Janeiro & 2012 \\
\hline & São Paulo & 2011 \\
\hline & Minas Gerais & 20--? \\
\hline & Espírito Santo & 2009 \\
\hline \multirow{3}{*}{ Sul } & Santa Catarina & 2014 \\
\hline & Paraná & 2008 \\
\hline & Rio Grande do Sul & 2009 \\
\hline
\end{tabular}

Fonte: Elaborado pelas autoras.

\section{Resultados e discussão}

\section{Abordagens da saúde e promoção da saúde}

A localização e a indicação de como o tema saúde deve ser abordado revela importantes pistas sobre como o tema tem sido encarado nas propostas curriculares do Brasil. Dentre as matrizes curriculares analisadas, 13 documentos explicitam em seu texto que a temática deve ser considerada, principalmente, junto aos $7^{\circ}$ e $8^{\circ}$ ano do ensino fundamental. 
Nestas classes, o tema se mostra alinhado com tópicos voltados aos seres vivos e corpo humano. Nesse sentido, poucas exceções podem ser encontradas. Uma delas é o documento do estado de Rondônia que não relaciona a temática a uma série específica, mas reproduz seu caráter interdisciplinar. Contudo, ao reportar os temas transversais e citá-la no tópico sobre educação ambiental, é na seção nomeada "Orientação sexual / prevenção e promoção à saúde" que ela ganha destaque (RONDÔNIA, 2013, p. 23). Apesar de ambos serem temas transversais distintos eles são colocados em conjunto.

De modo geral, os currículos de Ciências ao tratar da saúde como componente curricular enfatizam os tópicos de saúde sexual, reprodutiva e corpo humano. Tal questão é exemplificada no trecho a seguir: "Compreender as diferentes dimensões da reprodução humana e os métodos anticoncepcionais, valorizando o sexo seguro e a gravidez planejada" (PIAUÍ, 2013, p. 123). Ou seja, outras questões também relevantes ao cotidiano acabam sendo relegadas. Além disso, pouca é a relação estabelecida do tema saúde com outras disciplinas do currículo. Tratando-se de um tema transversal é evidente a sua potencialidade para que haja uma abordagem integrada com as demais disciplinas e também com outros temas transversais como, por exemplo, meio ambiente. No entanto, a relação mais proeminente incorporada é do tema saúde com o tema sexualidade.

Há uma dicotomia em relação à abordagem da saúde e à promoção da saúde. $\mathrm{O}$ viés progressista sinaliza para uma dimensão da promoção da saúde que valoriza a melhoria da qualidade de vida das populações baseada, principalmente, em políticas intersetoriais. Assim, a saúde é concebida como uma dimensão muito mais ampla do que aquela abarcada somente pelo setor da saúde e, consequentemente, ela não é dependente unicamente do condicionante biológico. A análise das propostas curriculares revelou que estes documentos tendem a propor um olhar sobre a saúde com a dimensão conservadora. Tal linha conservadora em relação à promoção da saúde se encarrega de responsabilizar o indivíduo e isentar o Estado no que diz respeito a saúde e seus determinantes (CZERESNIA, 2009).

Além disso, Moreira et al. (2009) sucinta as três principais concepções que são creditadas à saúde, a saber: (1) ausência de doença; (2) higienista/sanitarista; e, (3) biopsicossocial. O primeiro modelo é atribuído a uma visão de compreensão do corpo humano como estruturado por partes e que seu funcionamento é semelhante ao de uma máquina. A vertente higienista busca amparo nas descobertas do final do século XIX e início do século XX, quando se acreditava que a doença era devida sobretudo à presença de microrganismos e visava-se uma higienização massiva.

Em contrapartida, no modelo biopsicossocial sinalizado pela OMS, a partir da reunião realizada no período após a Segunda Guerra Mundial, a saúde é compreendida como produto de múltiplos determinantes (sociais, ambientais, genéticos, psicológicos, etc.). Nas propostas curriculares analisadas predomina o modelo de saúde como oposto ao de doença e/ou uma visão higienista a este respeito. "Reconhecer a necessidade e cuidados com a higiene pessoal, compreendendo as contribuições para a saúde e o bom convívio social” (GOIÁS, 20--?, p. 329, grifo nosso). "Reconhecer a necessidade da higiene corporal para preservar a saúde." (MATO GROSSO DO SUL, 2012, p. 285, grifo nosso).

Além disso, é marcante a presença de uma perspectiva biopsicossocial em relação à saúde e uma visão progressista em relação à promoção da saúde, conforme segue: "Compreender o corpo humano e sua saúde como um todo integrado por dimensões biológicas, afetivas e sociais, relacionando a prevenção de doenças e promoção de saúde das comunidades a políticas públicas 
adequadas” (PIAUÍ, 2013, p. 123). "Compreender a saúde como resultado do bem-estar físico, social, mental e cultural dos indivíduos (ESPÍRITO SANTO, 2009, p. 429).

Os Estados do Acre, Pernambuco e Paraná, ao tratarem da saúde, manifestam a intenção de formação de sujeitos capazes de interferir sobre agravos que atingem as respectivas regiões. "Favorecer o desenvolvimento de atitudes favoráveis de cuidado consigo mesmo e com os outros, a partir do conhecimento de práticas construtivas e de zelo com a saúde" (ACRE, 2010, p. 9, grifo nosso).

Compreender o organismo humano de forma sistêmica, interpretando diferentes relações e correlações, reconhecendo os fatores internos e externos que concorrem para a homeostasia, as manifestações e os modos de prevenção de doenças comuns e o papel da sociedade humana na preservação da saúde individual e coletiva (PERNAMBUCO, 2013, p. 31, grifo nosso).

Entretanto, o ideal de formação de indivíduos para a mitigação de agravos deve ser encarado não como algo despropositado. Pelo contrário, historicamente verifica-se nas políticas de prevenção e controle de agravos a materialização do fenômeno denominado culpabilização da vítima. Nesse sentido, o indivíduo é responsabilizado e a ele é delegada a responsabilidade sobre o controle e prevenção de doenças de forma pragmática. Não se consideram os agravos de saúde de forma holística e o Estado acaba por ser isento de suas responsabilidades (STOTZ, 1993).

Historicamente, os materiais educativos e informativos impressos apresentam uma perspectiva em que o leitor é mero depositário de informações e a ele é atribuída toda a responsabilidade sobre sua condição de saúde. Em estudos realizados no Brasil sobre materiais impressos sobre dengue e leishmanioses, verificou-se a presença de um discurso que pouco contribui para uma formação autônoma dos cidadãos (ASSIS; PIMENTA; SCHALL, 2013; LUZ et al., 2003). Assim, a escola, ao agregar essa mesma perspectiva no texto que orienta suas práticas, deixa de ser uma instituição que participa para a construção da cidadania.

Siatras e Koumaras (2013), ao analisarem os currículos de Ciências de dois países europeus em relação a temas sociocientíficos que integram o eixo saúde e ambiente, concluíram que é necessário pensar em uma formação que conjugue conhecimentos científicos e competências capazes de favorecer a reflexão sobre problemas cotidianos. Desta forma, se favorece a construção de uma sociedade mais equânime (SIATRAS; KOUMARAS, 2013). Assim, o modelo de ensino voltado somente ao conhecimento acadêmico ou à difusão de práticas de forma acrítica não atende mais as necessidades da sociedade atual. Ao lançar os olhos sobre os currículos de Ciências, reforçamos a importância de que se deva pensar não só sobre o conteúdo expresso, mas também sobre as indicações relacionadas e as ideologias que possivelmente podem estar vinculadas.

As recomendações reportadas pelas propostas curriculares, além de ideologias, apresentam implicações diretas para a prática. Como exemplo disso, os documentos dos estados de Rondônia e Bahia indicam que sejam realizadas atividades e associações com o setor da saúde.

[...] a escola deve criar estratégias que possam envolver toda sociedade nas questões que tratam da saúde pública [...]. Assim, se pressupõe a interse- 
ção da Educação com vários outros saberes, em especial com a área de Saúde (RONDÔNIA, 2013, p. 24, grifo nosso).

[...] a utilização de vídeos e a realização de palestras (com nutricionistas e agentes de saúde) contribuem, significativamente, para a compreensão dos conteúdos conceituais, procedimentais e atitudinais (BAHIA, 2018, p. 139, grifo nosso).

Segundo as matrizes curriculares, esses profissionais seriam capazes de informar a população sobre agravos e contribuir, assim, diretamente para a promoção da saúde. Contudo, esta é uma questão controversa. Na forma como está indicado nos textos analisados se expressa a ideia da educação para saúde difundida em meados do século XX. Segundo essa perspectiva para que o indivíduo alcançasse o estado pleno de bem-estar físico, mental e social era necessário que este fosse educado para a saúde (GAZZINELLI et al., 2005). Ou seja, nesta linha, a doença envolveria ou falta de informação ou um déficit cognitivo.

Alertamos para a necessidade de que a saúde seja percebida e tenha sua enunciação nos currículos escolares como um aspecto mais amplo e determinado por múltiplos fatores e não seja considerada com um bem de caráter puramente biológico. Além disso, advogar a favor deste conceito de saúde pressupõe fortalecer práticas educativas que visem à promoção da saúde e não simplesmente à prevenção de doenças.

\section{Doenças negligenciadas}

Em 2011, foi divulgada Nota Técnica do Instituto Oswaldo Cruz reportando a emergência de se colocar em pauta no Plano Brasil sem Miséria a questão das doenças negligenciadas. Dentre outras recomendações, o documento destacava a necessidade de inclusão do tema nos currículos do ensino básico (ARAÚJO-JORGE, 2011). Contudo, somente nove dos 24 documentos $(37,5 \%)$ recomendam que as doenças negligenciadas devam ser abordadas. O Quadro 2 evidencia as doenças citadas nas propostas curriculares analisadas.

A AIDS é o agravo mais retratado nos documentos $(n=6)$. A presença identificada em maior número pode ser atribuída à expansão do debate em torno do tema motivado pela epidemia a partir dos anos 1990 (PEREIRA; MONTEIRO, 2015). Segundo dados do Ministério da Saúde, entre 2007 a junho de 2015 foram registrados 93.260 casos de infecção de HIV no Brasil. (BOLETIM EPIDEMIOLÓGICO HIV AIDS, 2015). O número elevado indica a necessidade de reforço das políticas públicas específicas. Portanto, ao trazer para a sala de aula questões presentes na sociedade contribui-se para minimizar esta situação e para a construção da cidadania. "Compreendam os processos da fecundação e gravidez, reconhecendo o uso de preservativos como fator importante na preservação da saúde e prevenção das DST, especialmente da AIDS". (CEARÁ, 20--?, p. 6).

Dos 24 currículos analisados, quatro (Sergipe, Mato Grosso do Sul, Rio de Janeiro e Rio Grande do Sul) reportam a dengue como tema a ser considerado. O Brasil é um país onde o agravo é endêmico e deve-se "Compreender a relação existente entre o retorno de certas doenças como dengue e cólera e o cuidado individual, coletivo e governamental com o ambiente." (RIO DE JANEIRO, 2012, p. 7). 
Documentos de organismos internacionais como a Organização Mundial de Saúde (OMS) e Organização Pan-Americana da Saúde (OPAS) têm indicado a importância da temática no espaço escolar. Contudo, é necessário que transcenda a visão da escola como um local voltado ao controle social, direcionando-a para a formação cidadã. No Brasil, o boletim epidemiológico n. 44 (BOLETIM EPIDEMIOLÓGICO, 2015) indica 1.587 .080 possíveis casos de dengue no território nacional. Ao mesmo tempo, o principal vetor da dengue no Brasil, o mosquito Aedes aegypti é associado a duas outras doenças que se expandem largamente sobre o país, a Febre de chikungunya e a Febre pelo vírus Zika (BOLETIM EPIDEMIOLÓGICO, 2015), além da Febre amarela. Esses dados indicam a necessidade de que os currículos sejam atualizados frequentemente, sejam flexíveis, e que incentivem os docentes a recorrer a fontes de informação confiáveis para embasar suas práticas.

Embora as doenças negligenciadas constituam um tema de relevância para o contexto brasileiro, verificamos que a grande maioria dos currículos de Ciências não aborda a questão. Saúde e doenças não são produzidas e perpetuadas somente por questões biológicas. Assim sendo, seu domínio ultrapassa o setor da saúde e sua discussão deve permear outras áreas do conhecimento de modo interdisciplinar (BARATA, 2009). Dentre os currículos analisados, e que omitem o tema, chama a atenção o documento do estado de Pernambuco. O estado é o único que possui um programa específico para controle de sete doenças negligenciadas de interesse local (tracoma, doença de Chagas, hanseníase, filariose, esquistossomose, helmintíase e tuberculose). Nenhuma dessas doenças foi mencionada no documento e tampouco a parceria estratégica da disciplina de Ciências com profissionais do programa. O Projeto Sanar foi implantado em 2011 em 108 municípios definidos como prioritários (PERNAMBUCO, 2013). Evidencia-se, deste modo, o descompasso entre as políticas públicas e a expressão, mesmo velada, de que os problemas de saúde são de domínio do setor da saúde.

Outras doenças negligenciadas de importância no contexto nacional são lembradas em menor proporção. A Doença de Chagas e as Leishmanioses, por exemplo, foram reportadas somente em três matrizes curriculares (Sergipe, Mato Grosso do Sul e São Paulo). Já a leptospirose é apontada nos documentos de Mato Grosso do Sul e São Paulo. Ascaridíase, Filariose, Esquistossomose Malária e Hepatite A são mencionadas em apenas um currículo.

As doenças negligenciadas e a perspectiva de saúde presente nas propostas curriculares são sumarizadas no Quadro 2. Cabe destacar que muitos documentos relacionam mais de uma perspectiva de saúde. Desta forma, reporta-se o hibridismo destes documentos.

Quadro 2. Doenças citadas e a perspectiva de saúde apontada nas propostas curriculares analisadas

\begin{tabular}{|c|l|l|l|}
\hline \multirow{2}{*}{ Região } & \multicolumn{1}{|c|}{$\begin{array}{c}\text { Estado } \\
\text { Selecionado }\end{array}$} & $\begin{array}{c}\text { Doenças Negligenciadas } \\
\text { abordadas }\end{array}$ & \multicolumn{1}{|c|}{ Perspectiva de saúde presente } \\
\hline \multirow{5}{*}{ Norte } & Acre & Nenhuma & Fenômeno biopsicossocial \\
\cline { 2 - 5 } & Rondônia & Nenhuma & Fenômeno biopsicossocial e higienista. \\
\cline { 2 - 5 } & Tocantins & Nenhuma & Fenômeno biopsicossocial e higienista \\
\cline { 2 - 5 } & Amapá & Nenhuma & $\begin{array}{l}\text { Fenômeno Biopsicossocial como produ- } \\
\text { to da ausência de doença, com ênfase na } \\
\text { orientação à questão biológica e ambiental. }\end{array}$ \\
\cline { 2 - 4 } & Roraima & Nenhuma & $\begin{array}{l}\text { Fenômeno resultante da ausência de } \\
\text { doença. }\end{array}$ \\
\hline
\end{tabular}


Quadro 2. continuação

\begin{tabular}{|c|c|c|c|}
\hline Região & $\begin{array}{c}\text { Estado } \\
\text { Selecionado }\end{array}$ & \begin{tabular}{|c|}
$\begin{array}{c}\text { Doenças Negligenciadas } \\
\text { abordadas }\end{array}$ \\
\end{tabular} & Perspectiva de saúde presente \\
\hline \multirow{8}{*}{ Nordeste } & Pernambuco & Nenhuma & $\begin{array}{l}\text { Fenômeno biológico com destaque a } \\
\text { compreensão do corpo humano e seu } \\
\text { funcionamento. }\end{array}$ \\
\hline & Alagoas & Nenhuma & $\begin{array}{l}\text { Fenômeno biopsicossocial. Ênfase no } \\
\text { bem-estar social. }\end{array}$ \\
\hline & Bahia & Nenhuma & $\begin{array}{l}\text { Fenômeno visto como ausência de doen- } \\
\text { ças. }\end{array}$ \\
\hline & Piauí & AIDS & Fenômeno biopsicossocial. \\
\hline & Ceará & AIDS & $\begin{array}{l}\text { Fenômeno resultante da ausência de } \\
\text { doenças }\end{array}$ \\
\hline & Paraíba & $\begin{array}{l}\text { Indica o termo "doenças } \\
\text { negligenciadas de forma } \\
\text { genérica". }\end{array}$ & $\begin{array}{l}\text { Fenômeno resultante da ausência de } \\
\text { doença }\end{array}$ \\
\hline & Sergipe & $\begin{array}{l}\text { Hepatite A, Leptospirose, } \\
\text { Dengue; AIDS, Doença de } \\
\text { Chagas, Malária e Leishma- } \\
\text { niose }\end{array}$ & $\begin{array}{l}\text { Fenômeno resultante da ausência de doen- } \\
\text { ça e higienista. }\end{array}$ \\
\hline & Maranhão & Nenhuma & $\begin{array}{l}\text { Fenômeno resultante da ausência de } \\
\text { doença. }\end{array}$ \\
\hline \multirow{4}{*}{$\begin{array}{l}\text { Centro- } \\
\text { oeste }\end{array}$} & \begin{tabular}{|l|} 
Distrito \\
Federal \\
\end{tabular} & Nenhuma & $\begin{array}{l}\text { Fenômeno resultante da ausência de doen- } \\
\text { ça e sanitário. }\end{array}$ \\
\hline & Goiás & Nenhuma & $\begin{array}{l}\text { Fenômeno resultante da ausência de } \\
\text { doença. }\end{array}$ \\
\hline & Mato Grosso & Nenhuma & Fenômeno biopsicossocial. \\
\hline & $\begin{array}{l}\text { Mato Grosso } \\
\text { do Sul }\end{array}$ & $\begin{array}{l}\text { AIDS, Dengue, Doença de } \\
\text { Chagas, Leishmanioses e } \\
\text { Leptospirose }\end{array}$ & $\begin{array}{l}\text { Fenômeno resultante da ausência de } \\
\text { doença. }\end{array}$ \\
\hline \multirow{4}{*}{ Sudeste } & Rio de Janeiro & Dengue e Cólera & $\begin{array}{l}\text { Fenômeno resultante da ausência de } \\
\text { doença. }\end{array}$ \\
\hline & \begin{tabular}{|l|} 
Espírito \\
Santo \\
\end{tabular} & Nenhuma & $\begin{array}{l}\text { Fenômeno resultante da ausência de doen- } \\
\text { ça e biopsicossocial. }\end{array}$ \\
\hline & Minas Gerais & Nenhuma & $\begin{array}{l}\text { Fenômeno resultante da ausência de doen- } \\
\text { ça e biopsicossocial. }\end{array}$ \\
\hline & São Paulo & $\begin{array}{l}\text { Leishmaniose, Doença de } \\
\text { Chagas e Malária, Esquis- } \\
\text { tossomose, Filariose e } \\
\text { Ascaridíase }\end{array}$ & $\begin{array}{l}\text { Fenômeno resultante da ausência de } \\
\text { doença. }\end{array}$ \\
\hline \multirow{3}{*}{ Sul } & Paraná & Nenhuma & $\begin{array}{l}\text { Fenômeno resultante da ausência de } \\
\text { doença. }\end{array}$ \\
\hline & $\begin{array}{l}\text { Santa Cata- } \\
\text { rina }\end{array}$ & AIDS & $\begin{array}{l}\text { Fenômeno resultante da ausência de } \\
\text { doença. }\end{array}$ \\
\hline & $\begin{array}{l}\text { Rio Grande } \\
\text { do Sul }\end{array}$ & AIDS e Dengue & $\begin{array}{l}\text { Fenômeno resultante da ausência de } \\
\text { doença. }\end{array}$ \\
\hline
\end{tabular}

Fonte: Adaptado e ampliado de Assis e Araújo-Jorge (2014b). 
Em uma revisão bibliográfica sistemática que relacionou a ocorrência das doenças negligenciadas e as desigualdades socioeconômicas, os autores reportaram a indicação de quanto maior a escolarização menor a ocorrência das doenças negligenciadas. Segundos os autores, devido à maior escolarização, os indivíduos teriam maior autonomia na tomada de decisão em prol das ações de controle e prevenção às doenças negligenciadas, cientes dos seus direitos e deveres para a construção de uma sociedade mais equânime (HOUWELING et al., 2016).

Ponderamos que o acesso à escolarização apresenta ligação direta com o compromisso de construção de um cenário mais equânime. Para isso o ensino precisa resguardar o princípio libertador e romper com o viés que credita que o público escolar é mero depositário de informações. Assim, a escola tem reconhecido potencial para a redução de desigualdades e consequentemente mitigação das doenças negligenciadas. Contudo, cabe destacar que as práticas educativas e as orientações contidas nas propostas curriculares devem estimular as ações de promoção de saúde e não somente a prevenção de agravos balizados no binômio orientação/ação.

\section{Considerações finais}

Ao analisar os currículos de Ciências quanto à abordagem da promoção da saúde e das doenças negligenciadas emergem importantes contribuições para o ensino. Observamos que prevalece o modelo em que se considera a saúde como algo estritamente biológico ou centrado no conceito de saúde como ausência de doença, higienista, em que as práticas educativas visam unicamente o repasse de informações. Não se objetiva nesse modelo uma educação promotora da autonomia e cidadania, mas sim a indicação de hábitos e comportamentos.

Em relação às doenças negligenciadas, apenas uma pequena parcela dos documentos analisados considera a temática como ponto relevante. Dentre os agravos, uns são mais negligenciados que outros no âmbito do ensino de Ciências. Enquanto que a AIDS, que não é considerada doença negligenciada, é reportada na maior parte dos textos que mencionam o tema das doenças negligenciadas outros agravos, como as geohelmintoses, por exemplo, são poucos difundidos embora apresentem relevância no território nacional, principalmente em regiões com baixo acesso ao serviço de saneamento básico (FAVRE et al., 2009).

Por fim, sugerimos a ampliação do debate sobre a presença do tema das doenças negligenciadas e seus determinantes no ensino básico, a nível nacional, e que sejam incentivadas atividades de reconhecimento dos agravos à saúde de maior ou menor relevância para abordagem nos diferentes territórios. É preciso também haver sensibilização para a percepção de que a saúde é um fenômeno multicausal e como tal requer práticas integradas para sua promoção. Ainda em tempo, consideramos que a construção das propostas curriculares devem ser produtos de múltiplos debates que envolvam principalmente a sociedade e educadores. Somente por esta via será possível superar o modelo de ensino baseado em temas desconexos com a realidade.

\section{Agradecimentos}

As autoras agradecem à Coordenação de Aperfeiçoamento de Pessoal de Nível Superior (Capes) e à Fundação de Amparo à Pesquisa do Rio de Janeiro (FAPERJ). 
Assis, S. S.; Araujo-Jorge, T. C.

\section{Referências}

ACRE. Secretaria de Estado de Educação e Esporte. Orientações curriculares. [Rio Branco, 2010]. Disponível em: <http://www.see.ac.gov.br/portal/index.php/documentos-2/ category/2-orientacoes-curriculares>. Acesso em: 24 jan. 2018.

ALAGOAS. Secretaria do Estado da Educação e do Esporte. Referencial curricular da educação básica para as escolas públicas de Alagoas. Maceió, 2010.

ALVES-MAZZOT'TI, A. J.; GEWANDSZNAJDER, F. O método nas ciências naturais e sociais: pesquisa qualitativa e quantitativa. São Paulo: Pioneira, 1998.

ARAÚJO-JORGE, T. C. Embasamento técnico e sugestões para ações de controle das doenças da pobreza no Programa de Erradicação da Pobreza Extrema no Brasil. Rio de Janeiro: IOC, 2011. (Nota técnica n ${ }^{\circ}$ 1/2011/IOC/Fiocruz/Diretoria). Disponível em: <http://www.fiocruz.br/portalchagas/media/download.pdf>. Acesso em 25 jan. 2018.

ASSIS, S. S. Análise de livros didáticos, materiais impressos e das percepções e práticas dos professores e profissionais de saúde: subsídios para a estratégia integrada de prevenção e controle da dengue. 2012. 239 f. Dissertação (Mestrado em Ensino em Biociências e Saúde) - Instituto Oswaldo Cruz, Rio de Janeiro, 2012.

ASSIS, S. S.; ARAÚJO-JORGE, T. Doenças negligenciadas e o ensino de ciências: reflexões elaboradas a partir das propostas curriculares. Ensino, Saúde e Ambiente, Rio de Janeiro, v. 7 , n. 1, p. 1-11, 2014a.

As doenças negligenciadas e a promoção da saúde: possibilidades e limites para a articulação entre os currículos de ciências e o Programa Saúde na Escola (PSE). Revista da SBEnBio, Rio de Janeiro, n. 7, 2014b. Disponível em: <http://www.sbenbio.org.br/ wordpress/wp-content/uploads/2014/11/R0763-1.pdf>. Acesso em: 25 jan. 2018.

ASSIS, S. S.; PIMENTA, D. N.; SCHALL, V. T. Materiais impressos sobre dengue: análise e percepções de profissionais de saúde e educação. Revista Brasileira de Pesquisa em Educação em Ciências, Belo Horizonte, v. 13, n. 3, p. 25-51, 2013. Disponível em: <https://seer.ufmg.br/index.php/rbpec/article/view/2474/1874>. Acesso em: 25 jan. 2018.

BAHIA. Secretaria da Educação. Proposta curricular: $6^{\circ}$ ao $9^{\circ}$ ano: ciências da natureza: dos 11 aos 14 anos. Salvador, [2018]. Disponível em: < http:// escolas.educacao.ba.gov.br/ sites/default/files/private/midiateca/documentos/2016/5-proposta-curricular-6o-ao-9oano-ciencias-humanas-143-153.pdf>. Acesso em: 25 jan. 2018.

BARATA, R. B. Como e por que as desigualdades sociais fazem mal à saúde. Rio de Janeiro : Ed. Fiocruz, 2009.

BOGDAN, R.; BIKLEN, S. Investigação qualitativa em educação: uma introdução à teoria e aos métodos. Porto: Porto Editora, 1994.

BOLETIM EPIDEMIOLÓGICO. Brasília: Ministério da Saúde, v. 46, n. 44, 2015. Disponível em: <http://portalarquivos2.saude.gov.br/images/pdf/2015/ dezembro/23/2015-049---Dengue-SE-48---para-publica----o-21.12.15.pdf>. Acesso em: 25 jan. 2018. 
O que dizem as propostas curriculares do Brasil ...

BOLETIM EPIDEMIOLÓGICO HIV AIDS. Brasília: Ministério da Saúde, v. 4, n. 1, 2015. Disponível em: <http://www.aids.gov.br/es/node/57749>. Acesso em: 25 jan. 2018.

BRICEÑO-LEÓN, R. To prevent diseases of poverty or to overcome poverty?: when equity matters in research. In: MATLIN, S. (Ed.). Global forum update on research for health: poverty, equity and health research. London: Pro-Book, 2005. p. 30-32. Disponível em: $<$ http://announcementsfiles.cohred.org/gfhr_pub/assoc/s14810e/s14810e.pdf $>$. Acesso em: 25 jan. 2018.

BRITO, L. D.; FREITAS, D. O ensino de biologia e as possibilidades de construções curriculares pela comunidade escolar: o caso da disciplina biologia aplicada. In: TEIXEIRA, P. M. M.; RAZERA, J. C. C. (Org.). Ensino de ciências: pesquisas e pontos em discussão. Campinas: Komedi, 2009. p. 191-226.

CEARÁ. Secretaria da Educação. Plano curricular. Fortaleza: SEDUC, 20--?

CZERESNIA, D. O conceito de saúde e a diferença entre prevenção e promoção. In: CZERESNIA, D.; FREITAS, C. M. (Org.). Promoção da saúde: conceitos, reflexões e tendências. Rio de Janeiro: Editora Fiocruz, 2009. p. 43-57.

DEMO, P. Metodologia científica em ciências sociais. São Paulo: Atlas, 1995.

ESPÍRITO SANTO (Estado). Secretaria de Estado da Educação. Currículo básico: escola estadual: guia de implementação. Vitória, 2009. Disponível em: <https://selecao.es.gov.br/ novo/Arquivo/Download/1210>. Acesso em: 25 jan. 2018.

FAVRE, T. et al. A rationale for Schistosomiasis control in elementary schools of the rainforest zone of Pernambuco, Brazil. PLoS: Neglected Tropical Diseases, San Francisco, v. 3, n. 3, p. 1-8, 2009.

GAZZINELLI, M. F. et al. Educação em saúde: conhecimentos, representações e experiência da doença. Cadernos de Saúde Pública, Rio de Janeiro, v. 21, n. 1, p. 200-206, 2005. Disponível em: <https://doi.org/10.1590/S0102-311X2005000100022 >. Acesso em: 25 jan. 2018.

GOIÁS. Secretaria de Estado da Educação. Currículo referência da rede estadual de educação de Goiás: versão experimental. Goiânia, [20--?]. Disponível em: < http://portal. seduc.go.gov.br>. Acesso em: 25 jan. 2018.

GOODSON, I. F. Currículo, teoria e história. Petrópolis: Vozes, 1995.

HAN, X. et al. Protection motivation theory in predicting intention to engage in protective behaviors against Schistosomiasis among middle school students in rural China. PLoS:

Neglected Tropical Diseases, San Francisco, v. 8, n. 10, p. 1-10, 2014.

HOUWELING, T. A. J. et al. Socioeconomic inequalities in neglected tropical diseases: a systematic review. PLoS: Neglected Tropical Diseases, San Francisco, v. 10, n. 5, 2016.

KRASILCHIK, M. O professor e o currículo das ciências. São Paulo: EPU, 1987.

. Reformas e realidade o caso do ensino das ciências. São Paulo em Perspectiva, São Paulo, v. 14, p. 85-93, 2000. 
Assis, S. S.; Araujo-Jorge, T. C.

LESLEY, J. D. et al. Bihar's pioneering school-based deworming programme: lessons learned in deworming over 17 million Indian school-age children in one sustainable campaign. PLoS: Neglected Tropical Diseases, San Francisco, v. 9, n. 11, 2015.

LUZ, Z. M. P. et al. Evaluation of informative materials on leishmaniasis distributed in Brazil: criteria and basis for the production and improvement of health education materials. Cadernos de Saúde Pública, Rio de Janeiro, v. 19, n. 2, p. 561-9, 2003.

MARANHÃO. Secretaria de Estado da Educação. Diretrizes curriculares. 3. ed. São Luís, 2014.

MATO GROSSO (Estado). Secretaria de Estado de Educação. Orientações curriculares: área de ciências da natureza e matemática: educação básica. Cuiabá, 2010.

MATO GROSSO DO SUL. Secretaria de Estado de Educação. Referencial curricular da rede estadual de ensino: ensino fundamental. Campo Grande, 2012. Disponível em: <http://www.sed.ms.gov.br/>. Acesso em: 25 jan. 2014.

MINAS GERAIS. Secretaria de Estado de Educação. Proposta curricular: ciências: ensino fundamental. Belo Horizonte: SEED MG, 20--?

MINAYO, M. C. S. O desafio do conhecimento: pesquisa qualitativa em saúde. 12. ed. São Paulo: Hucitec, 2010.

MOHR, A. A educação em saúde na escola: panorama e questionamentos a partir de depoimentos de professores de ciências de Florianópolis. In: SELLES, S. E. et al. Ensino de biologia: histórias, saberes e práticas formativas. Uberlândia: EDUFU, 2009. p. 107-126.

MOREIRA, M. C. A. et al. A saúde no livro didático de ciências: um exercício de análise. In: ENCONTRO NACIONAL EM PESQUISA EM EDUCAÇÃO EM CIÊNCIAS, 7., 2009, Florianópolis. Anais... Florianópolis: ABPEC, 2009.

NEGLECTED tropical diseases: becoming less neglected. The Lancet, London, v. 383, n. 9925, p. 1269, 2014.

NICOLAY, B. et al. Understanding heterogeneity in the impact of national neglected tropical disease control programmes: evidence from school-based deworming in Kenya. PLoS:

Neglected Tropical Diseases, San Francisco, v. 9, n. 9, p. e0004108, 2015.

PARAÍBA. Secretaria de Estado da Educação. Diretrizes operacionais para o

funcionamento das escolas da rede estadual de ensino. João Pessoa, 2013. Disponível em: <http://static.paraiba.pb.gov.br/2012/12/Diretrizes-Operacionais-de-Ensino-2013paginado-na-grafica.pdf $>$. Acesso em: 25 jan. 2018.

PARANÁ (Estado). Secretaria de Estado da Educação. Diretrizes curriculares da educação básica: ciências. Curitiba, 2008. Disponível em: <http://www.educadores.diaadia. pr.gov.br/arquivos/File/diretrizes/dce_cien.pdf>. Acesso em: 24 jan. 2018.

PARDO, C. M. L. Concepto y medición de la pobreza. Revista Cubana de Salud Pública, La Habana, v. 33, n. 4, 2007.

PEREIRA, Z. M.; MONTEIRO, S. S. Gênero e sexualidade no ensino de ciências no Brasil: análise da produção científica recente. Contexto e Educação, Ijuí, v. 30, n. 95, p. 117-146, 
O que dizem as propostas curriculares do Brasil ...

2015. Disponível em: < https://www.revistas.unijui.edu.br/index.php/contextoeducacao/ article/view/3155/4767>. Acesso em: 25 jan. 2018.

PERNAMBUCO. Secretaria de Educação. Parâmetros para a educação básica do Estado de Pernambuco: parâmetros curriculares de ciências naturais: ensino fundamental. Recife, 2013. Disponível em: < http://www.educacao.pe.gov.br/portal/upload/galeria/4171/ ciencias_parametros_ef.pdf $>$. Acesso em: 24 jan. 2018.

PERNAMBUCO. Secretaria Estadual de Saúde. Programa Sanar: doenças negligenciadas. 2013. Disponível em: < http://portal.saude.pe.gov.br/programa/secretaria-executiva-devigilancia-em-saude/programa-sanar-doencas-negligenciadas>. Acesso em: 31 dez. 2018.

PIAUÍ. Secretaria de Estado da Educação. Matrizes disciplinares do ensino fundamental: quadro das disciplinas por ano / série. Teresina, 2013. Disponível em: <http://www.seduc. pi.gov.br/diretrizes/Gabinete/3/>. Acesso em: 25 jan. 2018.

PINHÃO, F.; MARTINS, I. O discurso sobre saúde e ambiente no livro didático de ciências brasileiro. Revista Electrónica de Enseñanza de las Ciencias, Vigo, v. 11, n. 2, p. 342364, 2012. Disponível em: <http://reec.uvigo.es/volumenes/volumen11/REEC_11_2_5_ ex595.pdf>. Acesso em: 12 dez. 2012.

RIO DE JANEIRO (Estado). Secretaria de Estado de Educação. Currículo mínimo: ciências e biologia. Rio de Janeiro, 2012. Disponível em: < http://www.rj.gov.br/web/ seeduc/exibeconteudo?article-id=759820>. Acesso em: 24 jan. 2014.

RIO GRANDE DO SUL. Secretaria da Educação. Lições do Rio Grande: ciências da natureza e suas tecnologias. Porto Alegre, 2009.

RONDÔNIA. Secretaria de Estado de Educação. Ensino fundamental: referencial curricular de Rondônia. Porto Velho, 2013. Disponível em: <http://www.seduc.ro.gov.br/ curriculo/wp-content/uploads/2013/02/ENSINO-FUNDAMENTAL1.pdf > . Acesso em: 25 jan. 2018.

RORAIMA. Secretaria de Estado da Educação e Desportos. Proposta da rede pública estadual para o ensino fundamental (ciências). Boa Vista, 2010.

SANTA CATARINA (Estado). Secretaria de Estado da Educação. Proposta curricular de Santa Catarina: formação integral na educação básica. Florianópolis, 2014. Disponível em: $<$ http:/ /www.propostacurricular.sed.sc.gov.br/site/Proposta_Curricular_final.pdf $>$. Acesso em: 25 jan. 2018.

SANTOS, W. L. P. Educação científica na perspectiva de letramento como prática social: funções, princípios e desafios. Revista Brasileira de Educação, Rio de Janeiro, v. 12, n. 36, p. 474-492, 2007. Disponível em: <https://doi.org/10.1590/S1413-24782007000300007>. Acesso em: 25 jan. 2018.

SÃO PAULO (Estado). Secretaria da Educação. Currículo do Estado de São Paulo: linguagens, códigos e suas tecnologias. São Paulo, 2011. Disponível em: <http://www. educacao.sp.gov.br/a2sitebox/arquivos/documentos/237.pdf>. Acesso em: 25 jan. 2018. 
Assis, S. S.; Araujo-Jorge, T. C.

SERGIPE. Secretaria de Estado da Educação. Referencial curricular: rede estadual de ensino de Sergipe. Aracaju, 2011.

SIATRAS, A.; KOUMARAS, P. Science education as public and social wealth: the notion of citizenship from a European perspective. In: ANNUAL MEETING OF THE AMERICAN EDUCATIONAL RESEARCH ASSOCIATION, 2013, São Francisco. Disponível em: <http:/ / files.eric.ed.gov/fulltext/ED543244.pdf>. Acesso em: 25 jan. 2018.

STOTZ, E. Enfoques sobre educação e saúde. In: VALLA, V.; STOTZ, E. (Org).

Participação popular, educação e saúde: teoria e prática. Rio de Janeiro: Relumé-Dumará, 1993. p. 11-22.

TOCANTINS (Estado). Secretaria de Estado da Educação e Cultura. Referencial curricular do ensino fundamental das escolas públicas do Estado do Tocantins: ensino fundamental do $1^{\circ}$ ao $9^{\circ}$ ano. 2. ed. Palmas, 2009.

WORLD HEALTH ORGANIZATION. Generic framework for control, elimination and eradication of neglected tropical diseases. Geneva, 2015. Disponível em: $<$ http:/ / www.who.int/neglected_diseases/resources/NTD_Generic_Framework_2015.pdf > . Acesso em: 25 jan. 2018.

Sustaining the drive to overcome the global impact of neglected tropical diseases: second WHO report on neglected diseases. Geneva, 2012.

WORLD HEALTH ORGANIZATION. Scientific Working Group. Report of the Scientific Working Group meeting on dengue: Geneva, 1-5 october, 2006. Geneva, 2007. (TDR/SWG/08). Disponível em: <http://www.who.int/tdr/publications/documents/ swg_dengue_2.pdf?ua=1 >. Acesso em: 24 jan. 2018.

YAZBEK, M. C. Pobreza no Brasil contemporâneo e formas de seu enfrentamento. Serviço Social \& Sociedade, São Paulo, n. 110, p. 288-322, 2012. Disponível em: < https://doi. org/10.1590/S0101-66282012000200005>. Acesso em: 25 jan. 2018.

Artigo recebido em 20/11/2016. Aceito em 01/10/2017.

Endereço para contato: Fundação Oswaldo Cruz, Instituto Oswaldo Cruz, Laboratório de Inovações em Terapias, Ensino e Bioprodutos (LITEB), Pavilhão Cardoso Fontes, Avenida Brasil, 4.365, Manguinhos, Rio de Janeiro, CEP 21045-900, RJ, Brasil. 